\title{
Editorial
}

J.M. Davies MSC MD FRCPC*, R.E. Priddy BSc MBBS FanzCA FRCPC $†$

\section{Structural aspects of anaesthetic care}

departmental). One procedural aspect of care, completion of the anaesthetic record, was noted, in addition to structural inadequacies (record format). Although not one of the mandates of the programme, no measures of outcome were detailed. Clark mentions development of a critical incident study that will allow comparison within one or among hospitals. (Critical incident studies may be a measure of either process or outcome.) ${ }^{16}$ Other important activities of ASPENS have included development of a newsletter, provision of telephone numbers for consultation with specialist anaesthetists and subspecialists (e.g., obstetrics, paediatrics), initiation of problem-oriented continuing medical education, standardization of a prototype anaesthetic and postoperative care record, and facilitation of quality assurance programmes.

On repeat review, 24 of the 30 problems found on first review had been corrected, but nine "new deficiences" were noted. This is not surprising. One of the recognized "hazards" of Quality Assurance activities is that fixing one thing may then lead to the need to fix something else. This is due to the close interrelationship of system components ("coupling") 16 and hence the importance of rechecking the effect of any changes ("closing the loop").

However, what is surprising is the nature of the problems. These problems were neither sophisticated nor unique, but rather common ones, and reported repeatedly in the literature. For example, in the ASPENS report, the anaesthetic record of 17 departments was "poor", with respect to layout and charting space. ${ }^{15}$ Similarly, in 1988, Bembridge and Bembridge reported their review of the 22 anaesthetic charts in use in the 40 hospitals of the Yorkshire Regional Health Authority. Just over $75 \%$ had a designated space for the patient's name, fewer than $50 \%$ had a heading to indicated details of "airway maintenance technique" and a similar number lacked space to write postoperative orders. ${ }^{17}$

The ASPENS report also indicated difficulties with staffing in the post-anaesthesia care units of seven hospitals, with inadequate numbers and no requirements for specific training. Twenty-five years ago, Kok and Mullan recommended that "anaesthetic recovery rooms should be provided in all future hospitals. In these rooms badrisk patients (could) be nursed by experienced nursing 
sisters, under the expert eye of the anaesthetists and surgeons," and that "qualified nurses" who had completed a "Diploma in Intensive Nursing Care" be "available for duty in recovery rooms." "1

Why do anaesthetists not seem to learn from the problems of others? They seem unable to correct repeatedly identified problems with respect to structure of care. (This is clearly not a problem specific to any one region. Kok and Mullan indicated that their suggestions, published in 1969, "were first made in 1958."11)

Three of the factors which may contribute to this inability include the nature of anaesthetists, anaesthetic regulatory bodies, and accreditation of hospitals.

\section{Anaesthetists}

The day-to-day work of an anaesthetist isolates the individual from other anaesthetists, apart from a weekly meeting, a few moments in the lounge or when taking over a case. Anaesthetists have in the past restricted themselves to the operating room and may have not even had their own department, but been members of a department of surgery. As well, anaesthetists are often regarded (by themselves and others) as secondary care-givers to patients of another physician. These factors all contribute to a potential lack of influence within the hospital.

One of the strengths of anaesthetists is that of getting on with things, through the expedition of common sense, ingenuity and good training. However, the ability to work quietly in the background, often by compromising or "just coping," is also a weakness. The implicit lesson that this imparts to others (including trainees) is of a group of highly trained professionals willing to tolerate suboptimal conditions, both for themselves and their patients.

\section{Regulatory bodies}

In Canada, three organizations contribute directly to the regulation of anaesthetic care. The Royal College of Physicians and Surgeons of Canada (RCPSC) is responsible for the initial training and certification of specialists. Beyond this time, RCPSC plays little role in the anaesthetic community, apart from the recently introduced and voluntary Maintenance of Competence programme. (This is in contrast to the Australian and New Zealand College of Anaesthetists, which has established detailed standards, for example, from "Privileges in anaesthesia"18 to "Monitoring during anaesthesia." 19 The existence of these standards means that individual hospital departments or state/provincial organizations do not have to "re-invent the wheel.")

The Canadian Anaesthetists' Society (CAS) has published guidelines to the practice of anaesthesia since 1977, thus making Canada the first country to do so. Through the Canadian Standards Association (CSA), Canada also "became the first country to have a published comprehensive standard for anaesthetic gas machines. ${ }^{20}$ However, no adherence to the guidelines from either of these bodies is mandatory, thus contributing to the conditions and equipment problems described by Clark.

As well as these three agencies, provincial bodies and/ or legislation may affect anaesthetic care, with each province being somewhat different. For example, the Medical Society of Nova Scotia has produced "Guidelines to the Practice of Anaesthesia." ${ }^{\prime \prime}$ In contrast, similar guidelines are not found in Alberta.

\section{Hospital accreditation}

There is, however, another national body which could have some influence on anaesthetic care. The Canadian Council on Health Facility Accreditation (CCHFA) carries out review of hospitals every one to four years (depending on the grading given), although review is mandatory only for teaching institutions. Furthermore, future trends in the process of accreditation may result in even more problems with (at least) anaesthetic equipment. For example, the 1992 accreditation standards required that the "Operating Suite and Postanesthetic Recovery Unit" have "space, equipment and supplies" which complied with the "appropriate regulations and guidelines."2l These included "those established by the facility, the Canadian Standards Association, (and) governmental regulatory bodies." ${ }^{21}$ However, the Draft Standards for 1995, entitled "A Client-Centred Accreditation Program,," ${ }^{22}$ are even less precise. The section on "Surgical Care" makes no mention of equipment, but lists, for example, "ways in which personal choice is encouraged and supported" and identification of "client/patient centred goals and expected results of care. ${ }^{22}$ A statement is included that "care is delivered in accordance with applicable legislative requirements, standards of practice and respective code of ethics of each profession involved in providing care. ${ }^{22}$ Evaluation of equipment is to be found in the section of "Environmental Management," Standard 7: "Processes ensure that the space, equipment and supplies comply with the applicable legislation, regulations and codes," including the Canadian Standards Association. ${ }^{22}$ Whilst being admirable in intent, details relevant to anaesthesia are difficult to discern.

As government, health care economists and patients become more and more demanding, anaesthetists need to be increasingly cognizant of the need to influence and control their own work-place. What, therefore, can anaesthetists do?

Perhaps the solution to these structural problems lies in the type of peer review programme exemplified by ASPENS - a quasi-autonomous non-governmental organization, that has an initiative, funding, and staffing. 
More importantly, it is a collective, a group of anaesthetists working together to improve their common lot, and also overcoming their intrinsic isolation.

Can ASPENS be improved, in terms of extent of the programme or efficiency? With respect to the extent of the programme, Clark states that ASPENS has been unable to provide advice about anaesthetic and monitoring equipment, although 30 "major deficiencies requiring action" were identified. ${ }^{15}$ Perhaps a lesson could be learned from Manitoba where two comprehensive reviews of anaesthetic machines have been carried out in the past 17 years. In 1977, Craig and Longmuir instituted a postal survey of "all 85 acute care health institutions, ${ }^{20}$ which reviewed whether anaesthetic machines conformed to the then newly developed standard for anaesthetic gas machines, Canadian Standards Association (CSA) Standard Z-168.3 "Continuous Flow Inhalation Anaesthetic Apparatus (Anaesthetic Machines) for Medical Use." Of 200 machines, only six were considered "already satisfactory." ${ }^{20}$ Two years later the authors had completed "direct on-site inspection and testing of every anaesthetic machine in the province." Of the 212 machines examined, only three were "already satisfactory." Through the Manitoba Health Services Commission, all deficiencies were corrected, by machine replacement, upgrading, or removal from service. For $\$ 280,000$ (in 1980) the goal was achieved of having "uniform minimum design and safety features for every anaesthetic machine in the hospitals of the province. ${ }^{20}$ In the spring of 1988, Friesen et al. initiated a second province-wide upgrading programme, with similar findings of "serious equipment deficiencies ... identified in both urban and rural health care facilities." ${ }^{23}$ Again, a "cost-effective means of implementing a province-wide upgrade of all hospital-based anaesthetic equipment" was effected. ${ }^{23}$

With respect to efficiency of ASPENS, we would suggest that the Planning and Directing Group consider linking each review to the CCHFA accreditation survey. This linkage would not be formalized, but linked only in timing and could be an unofficial prelude to the CCHFA site visit. The importance of the voluntary and peer review nature of ASPENS could thus be maintained. Time and effort would be saved on the part of the hospital and both ASPENS and CCHFA review teams, and the problems of outcome-orientated accreditation described above avoided. One area of strength of the draft CCHFA standards is the requirement for documentation of the "interprofessional care process," including before surgery, during anaesthesia, and postoperatively. ${ }^{22}$ In addition, deficiencies with respect to guidelines could be minimized, with the pre-accreditation process helping to eradicate any lack of knowledge, understanding or ability to change. The first Manitoba survey was initiated after institution of a new standard. ${ }^{20}$ Other guidelines were published in the next ten years, yet, as Friesen $e t$ al. stated, "these guidelines (were) not well followed."23

Anaesthetists should remember that guidelines can be used in a facilitative manner and their influence on unresponsive admininstrators should not be underestimated. This is particularly important given increasing financial constraints on medical practice, and the need to maintain quality of anaesthetic care. We would strongly encourage the institution of a programme similar to ASPENS in every province, ideally with national coordination.

\section{Le côté matériel de l'anesthésie}

Dès le début, les anesthésistes ont démontré leur ferme volonté de s'auto-évaluer. Ils se sont d'abord contentés de compte-rendu de résultats: les premiers décès causés par l'éther ont fait l'objet de communications en 1845 , en Angleterre et, en 1848, en Australie.' John Snow a poursuivi avec des observations qui traitaient de linfluence de l'anesthésie et de la chirurgie sur le devenir des malades. ${ }^{2,3}$ Depuis, les communications n'ont cessé d'affluer; certaines études ont été réalisées volontairement, ${ }^{4,5}$ d'autres imposées d'office par les législateurs et les experts, ${ }^{6,7}$ d'autres entreprises strictement pour des raisons médico-légales. ${ }^{8-10}$ Toutes étaient motivées par le désir d'accéder à une meilleure qualité et à une plus grand sécurité.

Mais ces études ne se limitaient pas à des discussions sur les techniques et les résultats thérapeutiques. Quelques-unes comprenaient des descriptions détaillées des aspects matériels de l'anesthésie avec mise en lumière des problèmes qu'on associait à des résultats incertains. ${ }^{11-13}$ D'autres ont même tenté d'évaluer globalement le système des soins anesthésiques en incluant des considérations sur des facteurs tels que la gestion et des recommandations en vue d'améliorations subséquentes. ${ }^{14}$

Ce mois-ci, Clarke nous présente un type différent de relevé intitulé "The anaesthetic services programme encompassing Nova Scotia (ASPENS) " qui sintéresse presque exclusivement aux aspects matériels de l'anesthésie. ${ }^{15}$ En sa qualité " d'anesthésiste-coordonateur " actuel du programme, il expose l'historique et les résultats d'un vaste programme d'évaluation collégiale destiné aux 
départements d'anesthésie. ${ }^{15}$ Initié par le Dr Emerson Moffitt, premier coordonateur du programme, son objectif consistait à améliorer la qualité de l'anesthésie en conformité avec les directives de la Société canadienne des anesthésistes, la Société médicale de la NouvelleÉcosse et Standards Canada. Entre 1987, année de l'institution du programme, et le milieu de 1993, 26 inspections ont été complétées dans 22 hôpitaux. Seuls trois hôpitaux de la province demeurent sur la liste d'attente.

L'enquête ASPENS constate des carences de personnel (tant en nombre qu'en qualifications), d'équipement, d'environnement et de gestion (intra- et interdépartementale). Du dossier, elle a évalué autant la tenue que le format. Comme les mesures de résultats ne faisaient pas partie des mandats du comité, elles n'ont pas été considérées. Clark mentionne la mise au point d'une évaluation des incidents graves qui permettra la comparaison infra- ou interhospitalière. (Ce type d'étude peut aussi bien mesurer les méthodes que les résultats). ${ }^{16}$ Une autre réalisation importante de l'ASPENS est constituée par la création d'un bulletin, l'accès aux numéros de téléphone d'anesthésistes experts pour fins de consultations, l'initiation de la formation médicale continue orientée vers des problèmes spécifiques, la standardisation d'un prototype de dossier anesthésique et postopératoire, et la mise en place de projets d'assurance de la qualité.

$\mathrm{Au}$ moment de la visite de contrôle, 24 des 30 problèmes rencontrés au cours de la première expertise avaient été corrigés, mais neuf nouvelles déficiences étaient apparues. Ceci n'a rien de surprenant. Avec l'assurance de la qualité, la correction d'un problème conduit souvent à l'apparition d'un autre. Ceci est dû à l'étroite relation qui existe entre les composantes du système; 16 de là l'importance de vérifier à nouveau les effets de toute modification.

Cependant, ce qui surprend, c'est la nature des problèmes. Les problèmes ne sont ni compliqués ni uniques, mais plutôt ordinaires et déjà décrits maintes fois dans la littérature. Par exemple, on note dans le rapport ASPENS que les dossiers anesthésiques de 17 départements était pauvres au regard de la disposition et de l'espace graphique. ${ }^{15}$ Les mêmes défauts étaient rapportés par Bembridge et Bembridge dans leur révision des dossiers de $\mathbf{4 0}$ hôpitaux du Yorkshire Regional Health Authority. Seulement un peu plus de $75 \%$ avaient un espace désigné pour l'inscription du nom du patient, moins de $50 \%$ possédaient une rubrique pour linscription des détails techniques concernant les voies aériennes et le même nombre n'avait pas l'espace requis pour l'inscription des ordonnances postopératoires. ${ }^{17}$

Le rapport ASPENS décrit dans la salle de réveil de sept hôpitaux des carences de personnel tant sur le nombre que la formation. Il y a 25 ans, Kok et Mullan re- commandaient que des salles de réveil soient implantées dans tous les hôpitaux. Dans celles-ci, les patients à risque devaient être suivis par des infirmières expérimentées sous la surveillance compétente des anesthésistes et des chirurgiens; le personnel devait être constitué d'infirmières titulaires d'un diplôme de nursing en soins intensifs. "

Pourquoi les anesthésistes ne profitent-ils pas de l'expérience des autres? Ils semblent incapables de corriger des problèmes déjà identifiés à maintes reprises lorsquil sont en rapport avec l'aspect matériel des soins. (Ce problème n'est sûrement pas unique pour une seule région, Kok et Mullan ayant montré que leurs suggestions, publiées in 1969, avaient déjà été proposées en $1958^{11}$ ).

Trois facteurs pourraient contribuer à cette incapacité: la nature des anesthésistes, les organismes de contrôle et l'agrément hospitalier.

\section{Les anesthésistes}

A l'exception de la réunion hebdomadaire, de la rencontre au salon ou de la prise en charge d'un cas débuté, le travail quotidien de l'anesthésiste lisole de ses collègues. Dans le passé, les anesthésistes ont été confinés à la salle d'opération. A certains endroits, le département d'anesthésie n'existait même pas et ils faisaient partie du département de chirurgie. Les anesthésistes sont de plus souvent considérés (par eux-mêmes et par les autres) comme des intervenants secondaires sur des patients traités par d'autres médecins. Ces facteurs peuvent contribuer à leur manque d'influence à l'intérieur de l'hôpital.

Un des points forts de l'anesthésiste est sa capacité d'agir, basée sur le bon sens, l'ingéniosité et la formation reçue. Cependant, son habileté à travailler calmement dans l'ombre, souvent en négociant mais aussi en s'adaptant, peut constituer une faiblesse. Les témoins (incluant ceux qui sont en formation) ont souvent limpression que les anesthésistes constituent un groupe de professionels de haute compétence qui acceptent des conditions sous-optimales aussi bien pour eux-mêmes que pour leurs patients.

\section{Les organismes de contrôle}

Au Canada trois organisations contributent directement au contrôle des soins en anesthésie. Le Collège royal des médecins et chirurgiens du Canada (CRMCC) est responsable de la formation originale et de la certification des spécialistes. Jusqu'à maintenant, en anesthésie, à l'exception du programme de maintien de la compétence, le CRMCC a joué un rôle effacé (la situation est très différente en Nouvelle-Zélande et en Australie où le collège des anesthésistes a établi des normes détaillées allant des privilèges d'exercice jusqu'au monitorage ${ }^{19}$ ). L'existence de ces normes signifie que les départements 
hospitaliers ou les organisations régionales n'ont pas besoin " de réinventer le roue ".

Grâce à la Société canadienne des anesthésistes (SCA) qui publie depuis 1977 un code d'exercice, le Canada est le premier pays à s'impliquer dans ce domaine. Par l'intermédiaire de Standards Canada, le Canada est aussi le premier pays à émettre des normes appliquées aux appareils d'anesthésie. ${ }^{20}$ Malheureusement, il n'est pas obligatoire d'adhérer aux directives de ces deux organismes, ce qui conduit a des problèmes de matériel et à des conditions telles que décrites par Clark.

Comme ces trois organismes, les associations provinciales et la législature peuvent influencer l'exercice de l'anesthésie de façon distincte dans chaque province. Par exemple, la Société médicale de la Nouvelle-Écosse a émis des directives sur l'exercice de l'anesthésie ${ }^{15}$ dont on ne retrouve pas de pendant en Alberta.

\section{L'agrément hospitalier}

Il existe un autre organisme national susceptible d'influencer l'anesthésie. Le Conseil canadien d'agrément des établissements de santé (CCAES) visite les hôpitaux à des intervalles d'une à quatre années (selon le classement antérieur), bien que cette visite ne soit obligatoire que pour les hôpitaux d'enseignement. Il est à noter que les tendances futures du processus d'agrément peuvent susciter encore plus de problèmes entre autres, du côté de l'équipement. Par exemple, les standards d'agrèment de 1992 énoncaient que les salles d'opération et les salles de réveil devaient avoir à leur disposition de l'espace, de l'équipement et des fournitures conformément aux directives et règlements appropriés. ${ }^{21}$ Ceux-ci incluaient les règlements de l'institution, de Standards Canada et des organismes de contrôle gouvernementaux. ${ }^{21}$ Cependant les standards projetés pour 1995 pour l'agrément orienté vers le patient ${ }^{22}$ sont encore moins précis. La section consacrée aux soins chirurgicaux ne mentionne pas l'équipement, mais produit une liste d'objectifs orientés vers le patient et de résultats anticipés. ${ }^{22}$ Il est énoncé que les soins sont dispensés en accord avec les préalables légaux applicables, les normes d'exercice et le code d'éthique propres à chacune des professions impliquées dans la distribution des soins. ${ }^{22}$ L'évaluation du matériel se retrouve dans la section de la gestion de l'environnement. La norme 7 stipule que le mécanisme de contrôle doit assurer un espace, du matériel et des fournitures conformes aux lois applicables, aux règlements et aux codes, incluant Standards Canada. ${ }^{22}$ Malgré ces intentions louables, les articles qui concernent l'anesthésie sont peu nombreux.

A mesure que les exigences du gouvernement, des économistes et des patients augmentent, les anesthésistes doivent reconnaître quil faut influencer et contrôler leur propre milieu de travail. Comment peuvent-ils y arriver?

La solution de ces problèmes structuraux réside dans le type de révision collégiale mis en valeur par ASPENS, une organisation non gouvernementale quasi-autonome qui possède linitiative, les fonds et le personnel. $\mathrm{Ce}$ qui est encore plus important, il s'agit d'une collectivité, d'un groupe d'anesthésistes qui travaillent ensemble pour améliorer le sort commun et mettre fin à leur isolation intrinsèque.

Le programme ASPENS peut-il s'améliorer en étendant son champ d'action et en augmentant son efficacité? Considérant son champ d'action, Clarke remarque qu'ASPENS n'a pas formulé des recommandations précises sur le matériel d'anesthésie et le monitorage bien que 30 défauts majeurs nécessitant correction aient été décelés. ${ }^{14}$ La réponse pourrait venir du Manitoba où deux inspections en profondeur des appareils d'anesthésie ont été réalisées sur une période de 17 ans. En 1977, Craig et Longmuir ont enquêté par la poste auprès des 85 établissements de soins aigus ${ }^{20}$ pour savoir si leurs appareils d'anesthésie étaient conformes aux normes nouvellement en vigueur, la norme Z-168.3 de Standards Canada. Sur 212 appareils, seulement six ont été considérés d'emblée comme satisfaisants. Grâce à la commission des services de santé du Manitoba, toutes les déficiences ont été corrigées par rehaussement ou retrait du service. Pour la somme de $280,000 \$$ (de 1980), on a atteint l'objectif proposé qui était de procurer des appareils d'anesthésie de conception moderne et possédant les dispositifs de sécurité essentiels à tous les hôpitaux de la province. ${ }^{20} \mathrm{Au}$ printemps de 1988, Friesen et al. ont initié à l'échelle provinciale un nouveau programme d'amélioration et sont arrivés aux mêmes conclusions: il existait des déficiences sérieuses aussi bien dans les milieux urbains que ruraux. ${ }^{23}$ Une fois de plus, on a remis à point tout le matériel anesthésique de la province. ${ }^{23}$

$\mathrm{Au}$ regard de l'efficacité, nous suggérons que le groupe de planification et de direction d'ASPENS coordonne ses activités avec l'inspection d'agrément de la CCAES. Cette relation ne devrait pas être formelle mais devrait concorder avec la visite d'agrément et constituer un prélude officieux à celle-ci. Il est important de maintenir la nature bénévole et collégiale d'ASPENS. Le centre hospitalier et les deux équipes, celle d'ASPENS et du CCAES épargneraient temps et efforts. On éviterait de plus les problèmes que provoque l'agrément orienté vers les résultats. La nécessité de documenter le processus des soins pendant les périodes préchirurgicale, anesthésique et postopératoire constitue un des standards projetés par le CCAES. ${ }^{22}$ De plus, on pourrait minimiser la déficience des normes d'exercice par le processus de pré-agrément et atténuer ainsi les effets du manque de connaissance, de compréhension ou de réceptivité aux changements. ${ }^{20}$ 
Diverses normes d'exercice ont été publiées au cours des dix dernières années et comme le notaient Friesen et al., ces normes ont été mal suivies. ${ }^{23}$

Les anesthésistes doivent se rappeler que les normes et directives d'exercice sont parfois suivies avec nonchalance et que leur impact sur les administrateurs réfractaires ne doit pas être sous-estimée. Les restrictions financières et de la nécessité de maintenir la qualité des soins ont une influence de plus en plus importante sur l'exercice de la médecine. Nous encourageons fortement la mise en place de programmes identiques à celui d'ASPENS dans chaque province, idéalement avec une coordination nationale.

\section{References}

1 Cooper MG. The first reported death associated with anaesthesia in Australia. Anaesth Intensive Care 1991; 19: 265-6.

2 Snow J. The Inhalation of the Vapour of Ether in Surgical Operations. London: John Churchill, 1847.

3 Snow J. On Chloroform and Other Anaesthetics: Their Action and Administration. London: John Churchill, 1858.

4 Halford FJ. A critique of intravenous anesthesia in war surgery. Anesthesiology 1943; 4: 47-8.

5 Edwards G, Morton HJV, Pask EA, Wylie WD. Deaths associated with anaesthesia: a report on 1000 cases. Anaesthesia 1956; 11: 194-200.

6 Holland $R$. Anaesthetic mortality in New South Wales. Br J Anaesth 1987; 59: 834-41.

7 Dovies JM, Campbell LA. Fatal air embolism during dental implant surgery: a report of three cases. Can J Anaesth 1990; 37: 112-21.

8 Gannon $K$. Mortality associated with anaesthesia. A case review study. Anaesthesia 1991; 46: 962-6.

9 McIntyre $R W$. Medico-legal consequences. Can J Anaesth 1991; 38: 1035-6.

10 Caplan RA, Ward RJ, Posner K, Cheney FW. Unexpected cardiac arrest during spinal anesthesia: a closed claims analysis of predisposing factors. Anesthesiology 1988; 68: 5-11.

11 Kok OVS, Mullan BS. Deaths associated with anaesthesia and surgery. A review of 1573 cases. Medical Proceedings 1969; 15: 31-5, 55-61, 76-82, 91-8.

12 Harrison GG. Death attributable to anaesthesia. A 10-year survey (1967-1976). Br J Anaesth 1978; 50: 1041-6.

13 Sennett GS. Factors contributing to a reduction in anaesthetic mortality. S Afr Med J 1981; 59: 37-41.

14 Eagle CJ, Davies JM, Reason J. Accident analysis of large-scale technological disasters applied to an anaesthetic complication. Can J Anaesth 1992; 39: 118-22.

15 Clark AJ. The Anaesthetic Services Programme Encompassing Nova Scotia (ASPENS). Can J Anaesth 1994; 41 : 716-24.

16 Davies JM. Critical incidents during anaesthesia. In: Hea- ley TEJ, Cohen PJ (Eds.). Wylie and Churchill-Davidson's A Practice of Anaesthesia, 6th ed. Sevenoaks, Kent: Edward Arnold, 1994 (in press).

17 Bembridge $M$, Bembridge JL. A survey of anaesthetic charts. Anaesthesia 1988; 43: 690-3.

18 Privileges in anaesthesia. Policy Document P 2 (1991). Melbourne, Victoria: Australian and New Zealand College of Anaesthetists, 1991.

19 Monitoring during anaesthesia. Policy Document P 18 (1990). Melbourne, Victoria: Australian and New Zealand College of Anaesthetists, 1990.

20 Craig DB, Longmuir J. Implementation of Canadian Standards Association Z168,3-M 1980 Anaesthetic Gas Machine Standard: the Manitoba experience. Can Anaesth Soc J 1980; 27: 504-9.

21 Acute Care Large Community and Teaching Hospitals 1992. Canadian Council on Health Facilities Accreditation. Ottawa, Ontario, 1990.

22 A Client-Centred Accreditation Program. Acute Care Proposed Standards for 1995 (Second Draft). Canadian Council on Health Facilities Accreditation. Ottawa, Ontario, 1994.

23 Friesen RM, Hatton G, Bjornson J. The upgrading and replacement of anaesthetic equipment: a provincial approach. Can J Anaesth 1990; 37: 889-95. 